\title{
Wound Treatment Using Growth Factors
}

\author{
Keisuke Okabe, Ruka Hayashi, Noriko Aramaki-Hattori, Yoshiaki Sakamoto, Kazuo Kishi \\ Department of Plastic and Reconstructive Surgery, Keio University, Tokyo, Japan. \\ Email: *kkishi@a7.keio.jp
}

Received April $2^{\text {nd }}, 2013$; revised May $4^{\text {th }}, 2013$; accepted May $12^{\text {th }}, 2013$

Copyright (C 2013 Keisuke Okabe et al. This is an open access article distributed under the Creative Commons Attribution License, which permits unrestricted use, distribution, and reproduction in any medium, provided the original work is properly cited.

\begin{abstract}
Treatment with growth factors is an effective method to promote wound healing. Several growth factors have been used in wound treatment, including basic fibroblast growth factor (bFGF) discovered in the 1970s, platelet-derived growth factor (PDGF), epidermal growth factor (EGF), and bone morphogenetic protein (BMP). In 2001, Kaken Pharmaceutical Co., Ltd. launched Fiblast Spray in Japan. This product is a topical formulation containing recombinant human bFGF. It is used to treat decubitus ulcers and skin ulcers (burn ulcers and leg ulcers). There is increasing interest in its stimulatory effect on wound healing and its inhibitory effect on scar formation. Wound healing is a physiological phenomenon that involves a complex series of integrated cellular and biochemical responses, characterized by phases of hemostasis, inflammation, proliferation, and maturation. However, much of its detailed mechanism remains unknown. There have been many basic research studies on wound healing using cells and experimental animals. If findings can be used from such studies and clinical practice, development of even more effective treatments might be possible.
\end{abstract}

Keywords: Wound Healing; Growth Factors; bFGF

\section{Development and Present State of Treatment Using Growth Factors}

\subsection{Basic Fibroblast Growth Factor}

In 1974, Gospodarowicz reported that a substance in bovine pituitary extract had growth stimulating activity in fibroblasts. He named the substance "fibroblast growth factor" (FGF) [1]. The complete amino acid sequences of the basic FGF (bFGF) and acidic FGF (aFGF) were determined in the 1980s. This information has enabled mass production of recombinant FGF. In 1990, Kaken Pharmaceutical (Tokyo, Japan) began a clinical trial of recombinant human bFGF (generic name: trafermin). A product named "Fiblast Spray" was launched in June 2001. Its indications include decubitus ulcers and skin ulcers (burn ulcers and leg ulcers). Several reports have also shown its therapeutic effects on periodontal disease and bone fractures. Presently, there are ongoing clinical trials for ulcers, periodontal disease, and bone fractures. A recombinant non-human bFGF is sold in China. It is a recombinant bovine bFGF used to promote wound healing.

\subsection{Platelet-Derived Growth Factor}

In 1974, Ross et al. reported on a platelet-secreted humo-

${ }^{*}$ Corresponding author. ral factor that stimulated the proliferation and migration of vascular smooth muscle cells. He later named the factor "platelet-derived growth factor" (PDGF) [2]. PDGF is a dimer composed of two polypeptide subunits, PDGF A-chain and PDGF B-chain. These subunits form three dimeric forms PDGF-AA, PDGF-AB, and PDGF-BB. PDGF-BB is sold in the US, Canada, Europe, and South Korea under the brand name of Regranex ${ }^{\circledR}$ gel. It is indicated for diabetic ulcers, but there is an ongoing US clinical trial in patients with periodontal disease.

\subsection{Epidermal Growth Factor}

In 1962, Cohen et al. reported on a protein, epidermal growth factor (EGF), found among nerve growth factors (NGFs) extracted from mouse submandibular gland [3]. EGF stimulates the proliferation of various cells, including glial cells, fibroblasts, and epithelium-derived cells such as keratinocytes. EGF is sold as a drug in South Korea and Belgium. It is used to treat diabetic ulcers in South Korea, and it is used as eye drops to treat corneal ulcers in Belgium.

\subsection{Bone Morphogenetic Protein}

In 1965, Urist reported that when demineralized bovine bone matrix was implanted into rat subcutaneous tissue, 
it induced ectopic bone formation. He named the causative protein "bone morphogenetic protein" (BMP) [4]. Many BMP isoforms have been cloned to date. In addition to induction of bone formation, BMP has been shown to have wide-ranging functions such as regulation of cell proliferation, cell differentiation, organogenesis, and apoptosis. The development is particularly advanced in BMP-2 and BMP-7 (osteogenic protein-1 (OP-1)). BMP-2 is sold in the US under the product name INFUSE bone graft. It is a bone growth stimulator for spinal fusion. In Europe, BMP-2 is sold under the product name Induct OS, which has been approved as an adjunct to standard care for open fractures. BMP-7 (OP-1) is sold in the US, Spain, and Australia under the brand name OP-1. It is used to treat long bone nonunion.

\section{Effectiveness of bFGF and Its Mechanism of Action}

\subsection{Wound Healing and bFGF}

In recent years, there has been an upward trend in the rates of skin ulcers associated with underlying diabetes or peripheral artery diseases. Thus, there is increasing interest in the treatment of skin ulcers. Although general knowledge of decubitus prevention is beginning to be disseminated, there are still a large number of patients with decubitus ulcers associated with aging. An important medical issue is how these intractable ulcers can be cured. In addition, there is an increasing public demand to address issues involving how to minimize scarring after surgery or traumatic injury and how to heal wounds without scarring. In other words, the utmost imperatives for plastic and reconstructive surgeons are "to treat wounds that are difficult to cure" and "to cure wounds without scarring." In order to fulfill the imperatives, we must integrate findings of wound healing and scar formation and appropriately treat each patient. Since approximately 10 years ago, bFGF have been used in clinical practice. The formulations have been demonstrated to partially resolve the aforementioned issues and have become a powerful weapon.

Wound healing is a process that involves a series of cellular and biochemical responses characterized by phases of hemostasis, inflammation, proliferation, and maturation. However, much of its detailed mechanism remains unknown, and research studies are actively being conducted on the role of bFGF.

Unlike the other 20 types of FGF family molecules, bFGF is expressed in a variety of tissues. It promotes cell division and plays wide-ranging roles such as in chemotasis, angiogenesis, and development. bFGF-knockout mice exhibit no prominent phenotypes under normal conditions. However, they have been reported to exhibit delayed healing of full thickness skin wounds compared with wild-type mice. In addition, the knockout mice showed reduced collagen deposition at the wound site and thicker scabs [5]. Local application of bFGF can promote dermal and epidermal repair [6-8]. These findings have shown that bFGF has unique effects on wound healing that cannot be duplicated by other members of the FGF family.

In the process of wound healing, it is essential to have the proliferation of various cells, including fibroblasts, vascular endothelial cells, and epidermal cells. Growth factors such as bFGF, EGF, VEGF, and PDGF play major roles in this process. Kanazawa et al. [9] blocked the effect of bFGF on fibroblast proliferation using mitomycin-C and examined bFGF-induced fibroblast migration in wound healing. They reported that bFGF promoted fibroblast migration in a dose-dependent manner via the PI3-kinase-Rac1-JNK pathway. Akasaka et al. [10] suggested that, in the remodeling phase of wound healing, bFGF might inhibit scar formation by induction of apoptosis in fibroblasts in granulation tissue.

\section{2. bFGF and Scar Formation}

In recent years, an increasing number of reports have been published on the anti-scarring effect of bFGF. It indicates growing interest on the effects of bFGF "to treat wounds that are difficult to cure" and "to cure wounds without scarring." There are reports in which bFGF significantly inhibited scar formation after burn injury and improved the quality of wound healing [1113]. Based on these results, the Japanese Society for Burn Injuries recommended bFGF as a drug to treat second degree burns in the 2009 Clinical Practice Guidelines for Burn Injuries.

Presently, bFGF is not covered by the Japanese national health insurance as treatment for non-ulcer wounds. However, bFGF has also been reported to be effective in treating incisional wounds. Ono et al. administered bFGF immediately after surgery in patients who had undergone resection of skin tumors. They reported that bFGF significantly inhibited scar formation [14]. There are also reports in which bFGF reduced preexisting scars. Eto et al. examined the effects of bFGF on fibroblasts from normal tissue and hypertrophic scar tissue. They observed markedly increased MMP-1 and HGF gene expression [15]. They found that when scar tissue was implanted in nude mice, bFGF promoted the resorption of scar tissue. This finding suggested that bFGF might be useful in the reduction of scar tissue in preexisting scars.

\section{Treatment with bFGF}

\subsection{Basic Methods of Use}

Fiblast Spray is recombinant human bFGF and is indi- 
cated for decubitus ulcers and skin ulcers (burn ulcers and leg ulcers). In these ulcers, bFGF promotes cell proliferation and migration as mentioned earlier, and it is thought to contribute to the formation of high quality, well-vascularized granulation tissue.

Fiblast Spray is prepared at the time of use by dissolving bFGF powder in a solution included in the package. The resulting solution is sprayed onto the affected area using the included nozzle. The affected area is cleaned by washing and removal of excess moisture. Subsequently, Fiblast Spray is sprayed on the area. The drug is allowed to be absorbed by waiting for 30 seconds after spraying, and a dressing is applied. The remaining Fiblast solution is stored in a cool, dark place at $10^{\circ} \mathrm{C}$ or less but freezing must be avoided. Fiblast Spray is recommended to be used within 2 weeks after bFGF powder is dissolved due to stability concerns. Since this drug stimulates cell proliferation, it is contraindicated in patients with malignant tumor or a history of malignant tumor at the application site.

\subsection{Side Effects}

The website of Kaken Pharmaceutical (http://fiblast.jp/ feat.html) states that there were a total of 729 cases at the time of drug approval, of which 11 cases $(1.51 \%)$ developed side effects. The side effects were mainly those associated with the drug administration site: irritation and pain in 7 cases (0.96\%), erythema in 3 cases $(0.41 \%)$, and pruritus in 3 cases $(0.41 \%)$. Administration of this drug resulted in abnormal changes in the laboratory test values in 41 of 729 cases (5.62\%). The abnormal changes were mainly ALT (GPT) elevation in 15 of 612 cases (2.45\%) and AST (GOT) elevation in 7 of 611 cases (1.15\%).

There were 3411 cases included in the Drug Use Investigation of the Post-marketing Surveillance. Side effects were observed in 125 of these cases (3.66\%) at the completion of re-examination (re-evaluation of safety and efficacy under the Japanese Pharmaceutical Affairs Law). The side effects were mainly excessive granulation tissue formation in 35 cases (1.03\%) and pain at the administration site in 8 cases $(0.23 \%)$.

\subsection{Application of Treatment Using bFGF}

1) Use of bFGF with ointment or wound dressing

When bFGF is to be used with an ointment or wound dressing, the wound site is cleaned by washing. bFGF is sprayed onto the affected area, and the area is allowed to stand for 30 seconds. Subsequently, a dressing is applied in a standard manner.

2) Use of bFGF with artificial dermis

The use of bFGF has been reported to shorten the healing period after artificial dermis application at the wound site, such as the fingertip. Sugamata et al. [16] used artificial dermis and Fiblast Spray in 6 cases of fingertip amputation and found that the shapes of the fingertips were satisfactorily reconstructed in all patients. They reported that the mean time until healing was 36.75 days for Ishikawa type I amputation, 26 days for oblique amputation at the lateral nail, and 28 days for nail bed defect.

The Kyoto University research group of Kawai et al. developed artificial dermis with controlled release of bFGF using a collagen sponge, which contained bFGFimpregnated gelatin microspheres [17]. They demonstrated its effectiveness in an animal model of wound healing $[18,19]$. In addition, they began an investigator-initiated clinical trial in May 2010 to examine the effects of this product in patients with intractable ulcers.

3) Use of bFGF with negative pressure wound therapy

In April 2010, treatment using the "V.A.C. ATS System" (KCI Inc.) became covered by the Japanese national health insurance. Thereafter a combination of bFGF formulation and negative pressure wound therapy has become widely used in Japan, and there have been many reports on its high effectiveness.

4) Expansion of indications to include bone fractures

Fibroblast growth factor receptor 1 (FGFR1) is one of the FGF receptors. It is known that mutations in the FGFR1 gene cause Pfeiffer syndrome, and mutations in the FGFR2 gene cause Apert syndrome, Crouzon syndrome, and Jackson-Weiss syndrome. Constitutively active mutations in the FGFR3 gene cause achondroplasia and type II thanatophoric dysplasia. These findings highlighted the importance of FGF bioactivity in the induction and regulation of the cartilage premordia.

In recent years, there have been reports on various animal experiments in which bFGF was locally administered to bone fracture sites and stimulated fracture healing. In in-vitro studies, bFGF has been reported to strongly simulate proliferation of undifferentiated osteoblast progenitor cells and bone marrow stromal cells.

Kawaguchi et al. [20] performed a multicenter clinical trial to investigate the effectiveness of bFGF on bone union after osteotomy. They injected a gelatin hydrogel with controlled release of bFGF into knee osteoarthritis patients during high tibial osteotomy. They examined subsequent bone union at the osteotomy sites. bFGF accelerated bone healing in a dose-dependent manner at the time point of 16 weeks after surgery. In addition, it shortened the time until bone union. Presently, they are conducting a phase II trial.

5) Expansion of indications to include periodontal disease

In recent years, much discussion has been held regarding the potential of cytokine therapy as a treatment of chronic periodontal disease, which causes loss of 
periodontal tissue. In mainly canine experimental models, bFGF administration has been shown to stimulate significant regeneration of periodontal tissue.

An early phase II trial began in 2001 to investigate the efficacy of bFGF to induce periodontal tissue regeneration and to investigate the safety. The double blinded trial involved dose response concurrent controls, including placebo, and was conducted in 13 nationwide institutions in Japan. Radiographic findings showed that local administration of bFGF induced significant new alveolar bone formation in 2- and 3-walled alveolar bone defects [21]. In 2005, a late phase II trial (dose-response trial) began involving 25 nationwide institutions. The trial showed that the drug containing $0.3 \%$ bFGF induced significant new alveolar bone formation [22].

\section{Conclusion}

Treatment with growth factors is beginning to gain world wide prevalence, mainly in plastic and reconstructive surgery. Ten years have passed since Fiblast Spray, a bFGF formation, was launched in Japan. This formulation has shown its stimulatory effect on wound healing and its inhibitory effect on scar formation. It is important to provide appropriate treatment for each patient based on the findings of basic research and clinical results.

\section{REFERENCES}

[1] D. Gospodarowicz, "Localisation of a Fibroblast Growth Factor and Its Effect Alone and with Hydrocortisone on 3T3 Cell Growth,” Nature, Vol. 249, 1974, pp. 123-127. doi:10.1038/249123a0

[2] R. Ross, J. Glomset, B. Kariya, et al., "A Platelet-Dependent Serum Factor That Stimulates the Proliferation of Arterial Smooth Muscle Cells in Vitro," Proceedings of the National Academy of Sciences of the United States of America, Vol. 71, No. 4, 1974, pp. 1207-1210. doi:10.1073/pnas.71.4.1207

[3] S. Cohen, "Isolation of a Mouse Submaxillary Gland Protein Accelerating Incisor Eruption and Eyelid Opening in the New-Born Animal,” Journal of Biological Chemistry, Vol. 237, 1962, pp. 1555-1562.

[4] M. R. Urist, "Bone: Formation by Auto Induction,” Science, Vol. 150, No. 3698, 1965, pp. 893-899. doi:10.1126/science.150.3698.893

[5] S. Ortega, M. Ittmann, S. H. Tsang, et al., "Neuronal Defects and Delayed Wound Healing in Mice Lacking Fibroblast Growth Factor 2,” Proceedings of the National Academy of Sciences of the United States of America, Vol. 95, No. 10, 1998, pp. 5672-5677. doi:10.1073/pnas.95.10.5672

[6] G. S. McGee, J. M. Davidson, A. Buckley, et al., "Recombinant Basic Fibroblast Growth Factor Accelerates Wound Healing,” Journal of Surgical Research, Vol. 45, No. 1, 1988, pp. 145-153. doi:10.1016/0022-4804(88)90034-0
[7] P. A. Hebda, C. K. Klingbeil, J. A. Abraham, et al., "Basic Fibroblast Growth Factor Stimulation of Epidermal Wound Healing in Pigs,” Journal of Investigative Dermatology, Vol. 95, 1990, pp. 626-631. doi:10.1111/1523-1747.ep12513528

[8] R. Tsuboi and D. B. Rifkin, "Recombinant Basic Fibroblast Growth Factor Stimulates Wound Healing in Healing-Impaired db/db Mice," Journal of Experimental Medicine, Vol. 172, No. 1, 1990, pp. 245-251. doi:10.1084/jem.172.1.245

[9] S. Kanazawa, T. Fujiwara, S. Matsuzaki, et al., "bFGF Regulates PI3-Kinase-Rac1-JNK Pathway and Promotes Fibroblast Migration in Wound Healing," PLOS ONE, Vol. 5, No. 8, 2010, Article ID: e12228. doi:10.1371/journal.pone.0012228

[10] Y. Akasaka, I. Ono, T. Kamiya, et al., "The Mechanisms Underlying Fibroblast Apoptosis Regulated by Growth Factors during Wound Healing," Journal of Pathology, Vol. 221, No. 3, 2010, pp. 285-299. doi:10.1002/path.2710

[11] S. Akita, K. Akino, T. Imaizumi, et al., "A Basic Fibroblast Growth Factor Improved the Quality of Skin Grafting in Burn Patients,” Burns, Vol. 31, No. 7, 2005, pp. 855-858. doi:10.1016/j.burns.2005.04.008

[12] S. Akita, K. Akino, T. Imaizumi, et al., "The Quality of Pediatric Burn Scars Is Improved by Early Administration of Basic Fibroblast Growth Factor,” Journal of Burn Care \& Research, Vol. 27, No. 3, 2006, pp. 333-338. doi:10.1097/01.BCR.0000216742.23127.7A

[13] T. Uemura, H. Watanabe and Y. Uemura, "Clinical Use of Trafermin (Fiblast Spray) in Pediatric Outpatients with Burn Injuries,” Japanese Journal of Burn Injuries, Vol. 32, No. 5, 2006, pp. 291-297.

[14] I. Ono, Y. Akasaka, R. Kikuchi, et al., "Basic Fibroblast Growth Factor Reduces Scar Formation in Acute Incisional Wounds," Wound Repair and Regeneration, Vol. 15, No. 5, 2007, pp. 617-623. doi:10.1111/j.1524-475X.2007.00293.x

[15] H. Eto, H. Suga, N. Aoi, et al., "Therapeutic Potential of Fibroblast Growth Factor-2 for Hypertrophic Scars: Upregulation of MMP-1 and HGF Expression," Laboratory Investigation, Vol. 92, No. 2, 2012, pp. 214-223. doi:10.1038/labinvest.2011.127

[16] A. Sugamata, N. Yoshizawa and S. Oyama, “Treatment of Fingertip Amputation Using a Combination of Artificial Dermis and bFGF Formulation," Progress in Medicine, Vol. 26, 2006, pp. 2731-2735.

[17] K. Kawai, S. Suzuki, Y. Tabata, et al., “Accelerated Tissue Regeneration through Incorporation of Basic Fibroblast Growth Factor-Impregnated Gelatin Microspheres into Artificial Dermis,” Biomaterials, Vol. 21, No. 5, 2000, pp. 489-499. doi:10.1016/S0142-9612(99)00207-0

[18] N. Kanda, N. Morimoto, S. Takemoto, et al., "Efficacy of Novel Collagen/Gelatin Scaffold with Sustained Release of Basic Fibroblast Growth Factor for Dermis-Like Tissue Regeneration,” Annals of Plastic Surgery, Vol. 69, No. 5, 2012, pp. 569-574.

[19] N. Kanda, N. Morimoto, A. A. Ayvazyan, et al., "Evaluation of a Novel Collagen-Gelatin Scaffold for Achieving 
the Sustained Release of Basic Fibroblast Growth Factor in a Diabetic Mouse Model," Journal of Tissue Engineering and Regenerative Medicine, 2012. doi:10.1002/term.1492

[20] H. Kawaguchi, S. Jinguchi, T. Izumi, et al., "Local Application of Recombinant Human Fibroblast Growth Factor-2 on Bone Repair: A Dose-Escalation Prospective Trial on Patients with Osteotomy," Journal of Orthopaedic Research, Vol. 25, No. 4, 2007, pp. 480-487. doi:10.1002/jor.20315
[21] M. Kitamura, K. Nakashima, Y. Kowashi, et al., "Periodontal Tissue Regeneration Using Fibroblast Growth Factor-2: Randomized Controlled Phase II Clinical Trial,” PLoS ONE, Vol. 3, No. 7, 2008, Article ID: e2611. doi:10.1371/journal.pone.0002611

[22] M. Kitamura, M. Akamatsu, M. Machigashira, et al., "FGF-2 Stimulates Periodontal Regeneration: Results of a Multi-Center Randomized Clinical Trial," Journal of Dental Research, Vol. 90, No. 1, 2011, pp. 35-40. doi:10.1177/0022034510384616 